\title{
Recent results on collectivity and correlations in heavy-ion collisions from ATLAS
}

\author{
Dominik Derendarz ${ }^{\dagger}$ (on behalf of the ATLAS Collaboration) \\ Institute of Nuclear Physics Polish Academy of Sciences \\ ul. Radzikowskiego 152 \\ 31-342 Kraków, Poland \\ E-mail: dominik.derendarz@cern.ch
}

\begin{abstract}
The measurement of the multi-particle cumulants with $0.47 \mathrm{nb}^{-1}$ of $\mathrm{Pb}+\mathrm{Pb}$ collisions at $\sqrt{s_{\mathrm{NN}}}=$ $5.02 \mathrm{TeV}$ with the ATLAS detector at the LHC is presented. To investigate the impact of fluctuations arising from intrinsic centrality or volume fluctuations, the cumulants measured in two different event classes used for centrality definition are compared, one based on the total transverse energy deposited in the Forward Calorimeter and the second based on the number of charged particles reconstructed at midrapidity. In the most central collisions significant differences between two events classes are observed, and they are also present in the mid-central collisions but with smaller magnitude. Two new measurements of heavy-flavor flow are also presented. First, the production of muons from heavy-flavor decays is measured in $\mathrm{Pb}+\mathrm{Pb}$ collisions at $\sqrt{s_{\mathrm{NN}}}=2.76 \mathrm{TeV}$ using $0.14 \mathrm{nb}^{-1}$ of data. Second, the elliptic flow of prompt and non-prompt $J / \psi$ is measured in the di-muon decay channel in $\mathrm{Pb}+\mathrm{Pb}$ collisions at $\sqrt{s_{\mathrm{NN}}}=5.02 \mathrm{TeV}$ with an integrated luminosity of $0.42 \mathrm{nb}^{-1}$. Both measurements provide evidence for the presence of heavy-quark flow in heavy-ion collisions.
\end{abstract}

7th Annual Conference on Large Hadron Collider Physics - LHCP2019

20-25 May, 2019

Puebla, Mexico

\footnotetext{
${ }^{*}$ Speaker.

${ }^{\dagger}$ This work was supported in part by the National Science Centre of Poland grant 2016/23/B/ST2/00702 and by PL-Grid Infrastructure.
} 


\section{Introduction}

One of the most powerful tools to study the properties of the quark-gluon plasma (QGP) created in relativistic heavy-ion collisions is the azimuthal anisotropy of produced particles. Typically the azimuthal distribution of charged particles is characterized by the Fourier decomposition of $\mathrm{d} N / \mathrm{d} \phi \propto 1+2 \Sigma_{n=1}^{\infty} v_{n} \cos n\left(\phi-\Phi_{n}\right)$, where $v_{n}$ and $\Phi_{n}$ represent the magnitude and the eventplane angle of the $n$-th order flow harmonic. It is understood that in high energy $A+A$ collisions $v_{n}$ coefficients carry information about the hydrodynamic medium response to the asymmetric initial conditions [1].

A well-established and widely-used technique to measure the $v_{n}$ harmonics relies on calculating cumulants $\left(c_{n}\{k\}\right)$ from the multi-particle correlations [2]. This method allows the study of event-by-event correlations among the magnitudes of two different order flow harmonics $v_{n}$ and $v_{m}$ via the 4-particle symmetric cumulants $\left(s c_{n, m}\{4\}\right)$ [3]. Similarly, the 3-particle asymmetric cumulant $\left(a c_{n}\{3\}\right)$ is sensitive to correlations involving both the magnitude and phase of two flow harmonics $v_{n}$ and $v_{2 n}$ [4]. Cumulants are often calculated for events with similar activity (event classes), defined using observables that are monotonically related to the particle multiplicity (such as charged particle tracks multiplicity, sum of transverse energy, etc.). Due to fluctuations in the particle production process, the centrality for events selected in different event classes can fluctuate from event-to-event. Recent measurements from the ATLAS experiment [5] show the impact of the centrality fluctuations on the cumulants, symmetric cumulants and asymmetric cumulants in the $\mathrm{Pb}+\mathrm{Pb}$ collisions.

The azimuthal anisotropy of the heavy quarks can provide valuable information about the QGP transport properties. Heavy quarks are primarily produced at the early stage of the collision and acquire azimuthal anisotropy through the interactions with the collectively expanding medium [6]. This proceedings contribution reports new ATLAS measurements of heavy-flavor quarks flow via prompt and non-prompt $J / \psi$ candidates and muons originating from semi-leptonic decays of heavy-quarks in $\mathrm{Pb}+\mathrm{Pb}$ collisions.

\section{Flow fluctuations in 5.02 $\mathrm{TeV} \mathrm{Pb}+\mathrm{Pb}$ collisions}

The standard observable used in the ATLAS experiment to quantify centrality of the heavyion collision is the transverse energy deposited in Forward Calorimeter $\left(\Sigma E_{\mathrm{T}}\right)$. The left panel of Figure 1 shows the correlation between $\Sigma E_{\mathrm{T}}$ and the number charged particle tracks $\left(N_{\mathrm{ch}}^{\mathrm{rec}}\right)$ reconstructed with the $p_{\mathrm{T}}>0.5 \mathrm{GeV}$ and $|\eta|<2.5$. The linear correlation between these two quantities indicate that they are both sensitive to the number of particles created in the collision. However events with the same $\Sigma E_{\mathrm{T}}$ could have significant fluctuations in $N_{\mathrm{ch}}^{\mathrm{rec}}$ and the other way around, events with fixed $N_{\mathrm{ch}}^{\mathrm{rec}}$ could have different values of $\Sigma E_{\mathrm{T}}$. The mean and root-mean-square values of $N_{\mathrm{ch}}^{\mathrm{rec}}\left(\Sigma E_{\mathrm{T}}\right)$ calculated in narrow bins of $\Sigma E_{\mathrm{T}}\left(N_{\mathrm{ch}}^{\mathrm{rec}}\right)$ are shown in the middle (left) panel of Figure 1. A linear relation between $\left\langle N_{\mathrm{ch}}^{\text {rec }}\right\rangle$ and $\Sigma E_{\mathrm{T}}$ is observed over entire $\Sigma E_{\mathrm{T}}$ range. Same relation in seen when looking at the $\left\langle\Sigma E_{\mathrm{T}}\right\rangle$ in narrow bins of $N_{\mathrm{ch}}^{\mathrm{rec}}$ until the most central collisions where $\left\langle\Sigma E_{\mathrm{T}}\right\rangle$ deviates from the linear relation to $N_{\mathrm{ch}}^{\mathrm{rec}}$. This suggests that the true centrality distribution is more smeared for the events with the same $N_{\mathrm{ch}}^{\mathrm{rec}}$ than for events with the same $\Sigma E_{\mathrm{T}}$, and a reference event class based on $N_{\mathrm{ch}}^{\mathrm{rec}}$ may have different centrality fluctuations than the event class based on 

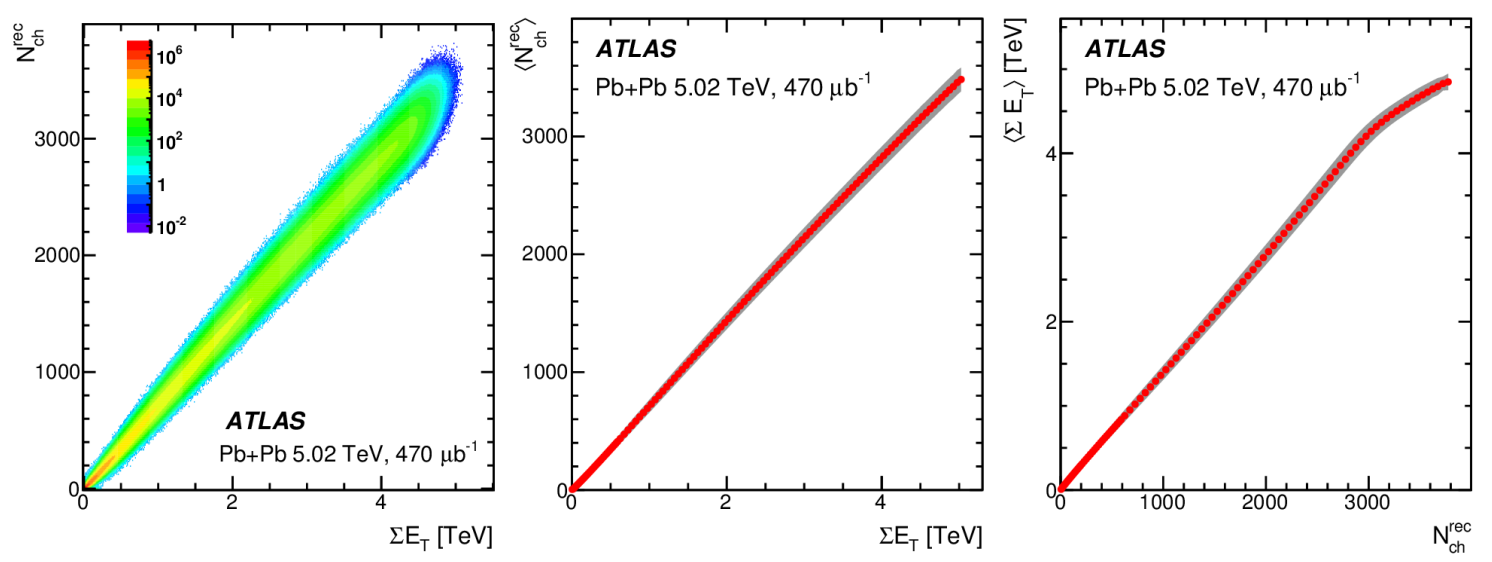

Figure 1: Correlation between $N_{\mathrm{ch}}^{\mathrm{rec}}$ and $\Sigma E_{\mathrm{T}}$ in the $\mathrm{Pb}+\mathrm{Pb}$ collisions at $\sqrt{s_{\mathrm{NN}}}=5.02$ (left). The mean values of $N_{\mathrm{ch}}^{\text {rec }}$ calculated in narrow slices of $\Sigma E_{\mathrm{T}}$ (middle) and the mean values of $\Sigma E_{\mathrm{T}}$ calculated in narrow slices of $N_{\mathrm{ch}}^{\mathrm{rec}}$ (right). Shaded bands show root-mean-square of either $N_{\mathrm{ch}}^{\mathrm{rec}}$ or $\Sigma E_{\mathrm{T}}$ [7].

$\Sigma E_{\mathrm{T}}$. The influence of centrality fluctuations on the measurement of flow harmonics is investigated by comparing cumulants calculated using $\Sigma E_{\mathrm{T}}$-based reference event class and $N_{\mathrm{ch}}^{\text {rec }}$-based event class.

Figure 2 shows the ratios of $v_{n}\{2\}$ obtained from 2-particle cumulants in two event classes. The contribution of non-flow correlation is reduced by the requirement of pseudorapidity gap of at least $|\Delta \eta|>2$ between two particles. In order to properly calculate the ratio, $v_{n}\{2\}$ values obtained in the narrow bins of $N_{\mathrm{ch}}^{\mathrm{rec}}\left(v_{n}\left\{2, N_{\mathrm{ch}}^{\mathrm{rec}}\right\}\right)$ are mapped to the $\left\langle\Sigma E_{\mathrm{T}}\right\rangle$ and similarly $v_{n}\{2\}$ values calculated in the narrow bins of $\Sigma E_{\mathrm{T}}\left(v_{n}\left\{2, \Sigma E_{\mathrm{T}}\right\}\right)$ are mapped to the $\left\langle N_{\mathrm{ch}}^{\text {rec }}\right\rangle$. The results are then plotted as a function of $\left\langle\Sigma E_{\mathrm{T}}\right\rangle$ scaled by $4.1 \mathrm{TeV}$ (top row) and as a function of $\left\langle N_{\mathrm{ch}}^{\mathrm{rec}}\right\rangle$ scaled by 2800 (bottom row). Values used for scaling indicate where $\Sigma E_{\mathrm{T}}$ and $N_{\mathrm{ch}}^{\text {rec }}$ distributions start to decrease sharply ("knee" of the centrality distribution) and the underlying centrality fluctuations are expected to be stronger. Such a representation of the $x$-axis gives more natural comparison of the overlap region in $\mathrm{Pb}+\mathrm{Pb}$ collisions and better comparison between the two event activity observables. The ratios shown as a function of $\left\langle\Sigma E_{\mathrm{T}}\right\rangle /(4.1 \mathrm{TeV})$ are consistent with unity for $v_{3}$ and $v_{4}$, but for the most central collisions $v_{2}$ shows a few percent deviation. This result suggests that events selected in narrow bins of $N_{\mathrm{ch}}^{\text {rec }}$ have slightly higher $v_{2}$ than events selected in narrow bins of $\Sigma E_{\mathrm{T}}$ when both ensembles are matched to the same $\left\langle\Sigma E_{\mathrm{T}}\right\rangle$. The ratios plotted as a function of $\left\langle N_{\mathrm{ch}}^{\mathrm{rec}}\right\rangle / 2800$ shows even more deviations from unity that are also present in $v_{3}$ and $v_{4}$. This behaviour is consistent with the hypothesis that $N_{\mathrm{ch}}^{\mathrm{rec}}$ have poorer centrality resolution and contains more events from less central collisions where events have larger $v_{n}$.

Figure 3 shows the direct comparison of normalized 4-particle cumulants $\left(n c_{n}\{4\}\right)$ obtained with the two reference event classes for particles in the range $1.5<p_{\mathrm{T}}<5.0 \mathrm{GeV}$. The advantage of multi-particle cumulants normalized by the average value of $c_{n}\{2\}$ is that the $p_{\mathrm{T}}$ dependence is canceled out and $n c_{n}\{k\}$ directly reflects probability density distribution of $v_{n}$ harmonic $\left(p\left(v_{n}\right)\right)$. The $n c_{2}\left\{4, \Sigma E_{\mathrm{T}}\right\}$ and $n c_{2}\left\{4, N_{\mathrm{ch}}^{\text {rec }}\right\}$ values are negative for the most of the $\Sigma E_{\mathrm{T}}$ range. In the most central collisions both cumulants change sign, reach positive maximum and then decrease to zero again. The normalized cumulants for $v_{3}$ harmonic are negative over the entire $\Sigma E_{\mathrm{T}}$ range and 

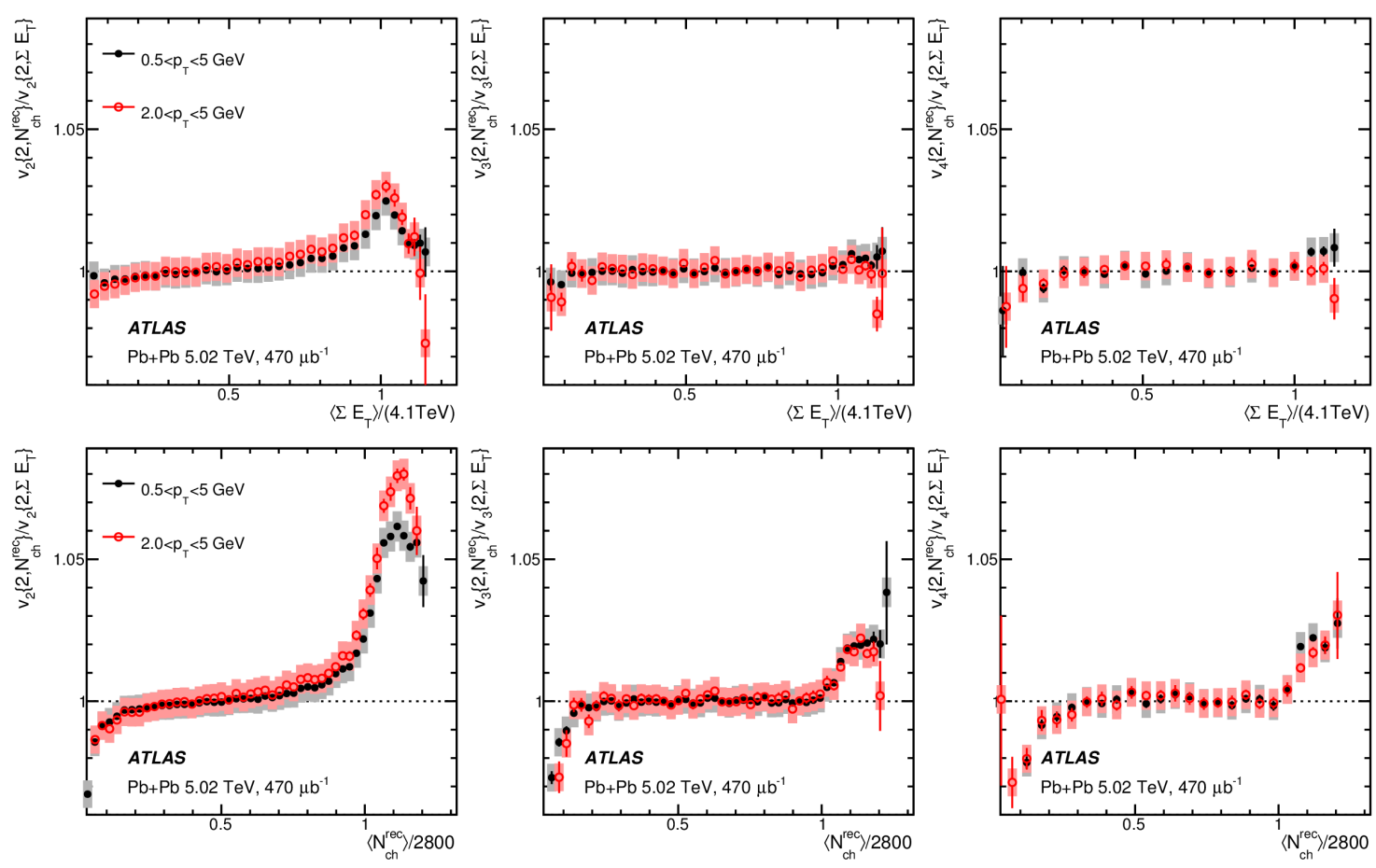

Figure 2: Ratio of flow harmonics measured in $\mathrm{Pb}+\mathrm{Pb}$ collisions at $\sqrt{s_{\mathrm{NN}}}=5.02$ using two event class definitions based on the $N_{\mathrm{ch}}^{\mathrm{rec}}$ and $\Sigma E_{\mathrm{T}}$ shown as a function of $\left\langle\Sigma E_{\mathrm{T}}\right\rangle$ (top) and $\left\langle N_{\mathrm{ch}}^{\mathrm{rec}}\right\rangle$ (bottom). Ratios are shown for the $v_{2}$ (left), $v_{3}$ (middle) and $v_{4}$ (right) harmonics. The error bars and shaded boxes represent the statistical and systematic uncertainties, respectively [7].
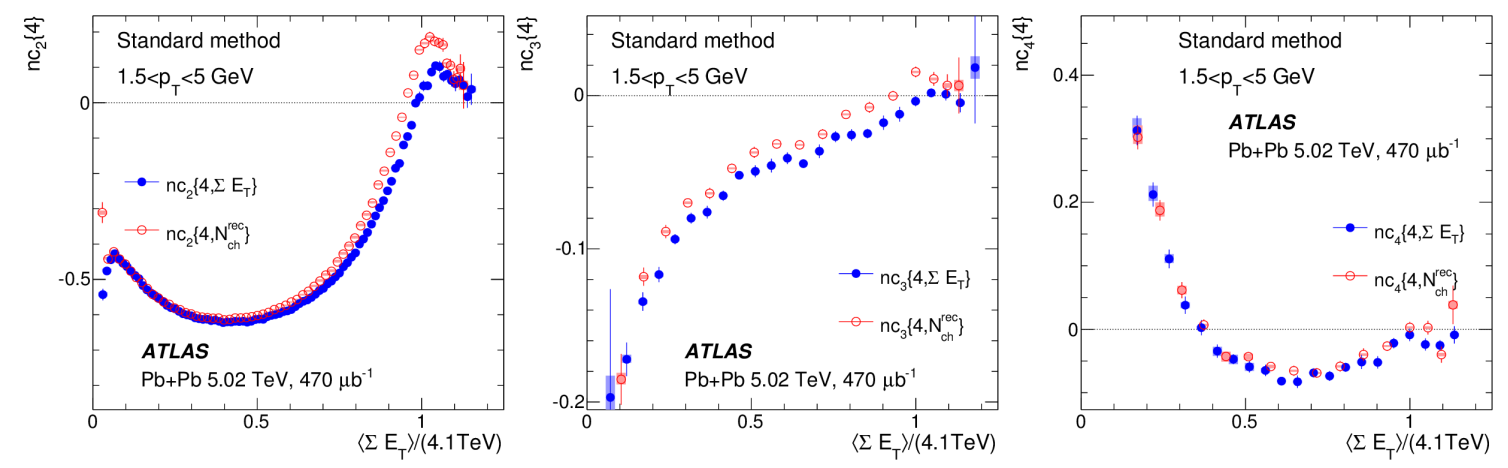

Figure 3: Comparison of $n c_{2}\{4\}$ (left), $n c_{3}\{4\}$ (middle) and $n c_{4}\{4\}$ (right) cumulants measured in $\mathrm{Pb}+\mathrm{Pb}$ collisions at $\sqrt{s_{\mathrm{NN}}}=5.02$ using two event class definitions and shown as a function of $\left\langle\Sigma E_{\mathrm{T}}\right\rangle$. The error bars and shaded boxes represent the statistical and systematic uncertainties, respectively [7]. 
approach zero in central collisions. The $n c_{4}\left\{4, \Sigma E_{\mathrm{T}} \mid N_{\mathrm{ch}}^{\mathrm{rec}}\right\}$ values change sign from positive in peripheral events to negative in the central events. Studies performed with the Glauber model [8] show that $c_{n}\{4\}$ is always negative when the reference event class is defined using the number of participating nucleons $N_{\text {part }}$ or the collision impact parameter [9]. Positive values of $c_{n}\{4\}$ obtained when a reference class is based on multiplicity of final state particles (both $N_{\mathrm{ch}}^{\mathrm{rec}}$ and $\Sigma E_{\mathrm{T}}$ ) could be explained by the non-Gaussian shape of $p\left(v_{n}\right)$ [10], but the impact of the centrality fluctuations is also seen in the higher values of the $n c_{2}\left\{4, N_{\mathrm{ch}}^{\mathrm{rec}}\right\}$. The normalized cumulants for $v_{2}$ and $v_{3}$ show significant differences between the two reference event classes, while the difference is much smaller for $v_{4}$. This suggests that the centrality fluctuations are potentially also important in mid-central collisions.

The impact of the centrality fluctuation on the normalized symmetric and asymmetric cumulants is also studied. Figure 4 shows that the $n s c_{2,3}\left\{4, N_{\mathrm{ch}}^{\mathrm{rec}}\right\}$ are significantly larger than the respective $n s c_{2,3}\left\{4, \Sigma E_{\mathrm{T}}\right\}$ over a broad centrality range, not only in most central collisions. In contrary to the normalized cumulants of single harmonics, where for each harmonic significant differences were observed for two reference classes, only $n s c_{2,3}\left\{4, N_{\mathrm{ch}}^{\mathrm{rec}}\right\}$ and $n s c_{2,3}\left\{4, \Sigma E_{\mathrm{T}}\right\}$ are significantly different. Other two cumulants, sensitive to the correlation between $v_{2}$ and $v_{4}$, are similar when calculated using the two reference event classes.
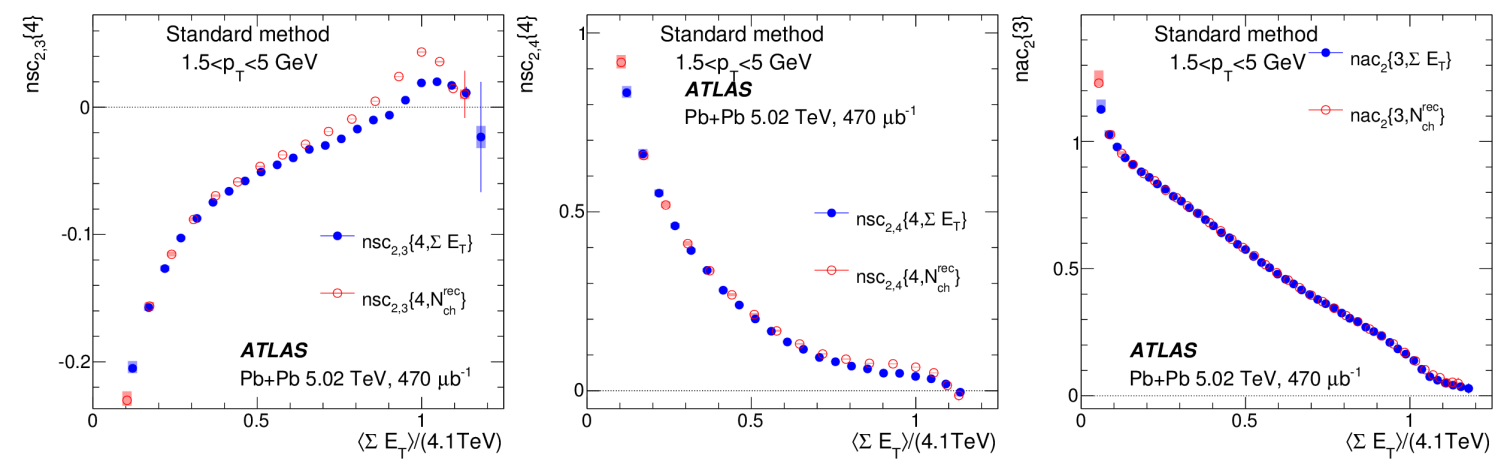

Figure 4: Comparison of $n s c_{2,3}\{4\}$ (left), $n s c_{2,4}\{4\}$ (middle) and $n a c_{2}\{3\}$ (right) measured in $\mathrm{Pb}+\mathrm{Pb}$ collisions at $\sqrt{s_{\mathrm{NN}}}=5.02$ using two event class definitions and shown as a function of $\left\langle\Sigma E_{\mathrm{T}}\right\rangle$. The error bars and shaded boxes represent the statistical and systematic uncertainties, respectively [7].

\section{Heavy-flavor flow in 2.76 $\mathrm{TeV}$ and 5.02 $\mathrm{TeV} \mathrm{Pb}+\mathrm{Pb}$ collisions}

In the early stages of the hadronic collision, charm $(c)$ and bottom $(b)$ quarks are produced via hard scatterings. As a result of their interactions with the collectively expanding medium, the heavy-quarks may acquire an azimuthal anisotropy. Measurements of the heavy-quark azimuthal anisotropy in $\mathrm{Pb}+\mathrm{Pb}$ collisions at the LHC can provide valuable constraints on the QGP transport parameters [13].

Figure 5 shows $v_{2}$ and $v_{3}$ of heavy-flavor muons from semi-leptonic decays of heavy-quarks in five centrality intervals measured as a function of $p_{\mathrm{T}}$. Heavy-flavor muons are distinguished from background muons using a "momentum-imbalance" variable that compares the momenta of the muons measured in the inner detector and muon spectrometer. Flow harmonics are measured 

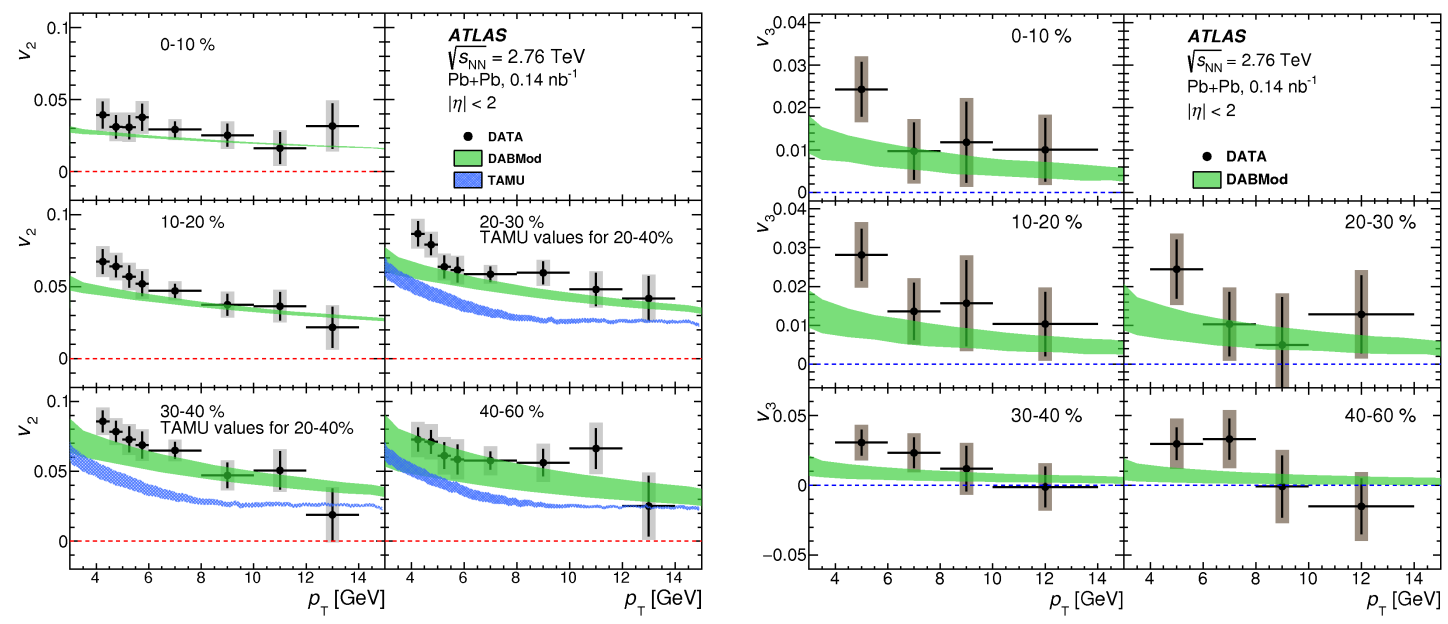

Figure 5: Comparison of the heavy-flavor muon $v_{2}$ (right) and $v_{3}$ (left) measured in $\mathrm{Pb}+\mathrm{Pb}$ collisions at $\sqrt{s_{\mathrm{NN}}}=2.76$ with calculations from TAMU and DABMod models [11]. For the data, the error bars and shaded bands represent statistical and total uncertainties, respectively. For the model calculations bands represent theoretical systematic uncertainties.

by evaluating the yields differentially relative to the $\Phi_{n}$ plane, corrected for the jet bias caused by the back-to-back recoil jet that is usually associated to the signal muon. In all centrality classes, significantly non-zero $v_{2}$ is observed for muons in the range $4<p_{\mathrm{T}}<12 \mathrm{GeV}$. The $p_{\mathrm{T}}$ dependence of $v_{2}$ for heavy-flavor muons is similar to those of charged hadrons $v_{2}$, with the characteristic drop at high $p_{\mathrm{T}}$, but smaller in magnitude. The $v_{3}$ signal is smaller than the $v_{2}$ and shows a weaker variation over centrality. Experimental data are also compared to prediction from TAMU (transport of the heavy-flavor within the QGP) [14] and DABMod (energy-loss of heavy-flavor in the QGP) [15] models. DABMod gives better a description of $v_{2}$ than TAMU probably due to implementation of the event-by-event fluctuations into the model. For both $v_{2}$ and $v_{3}$ DABMod values are smaller than the measured values but become consistent at higher $p_{\mathrm{T}}$.
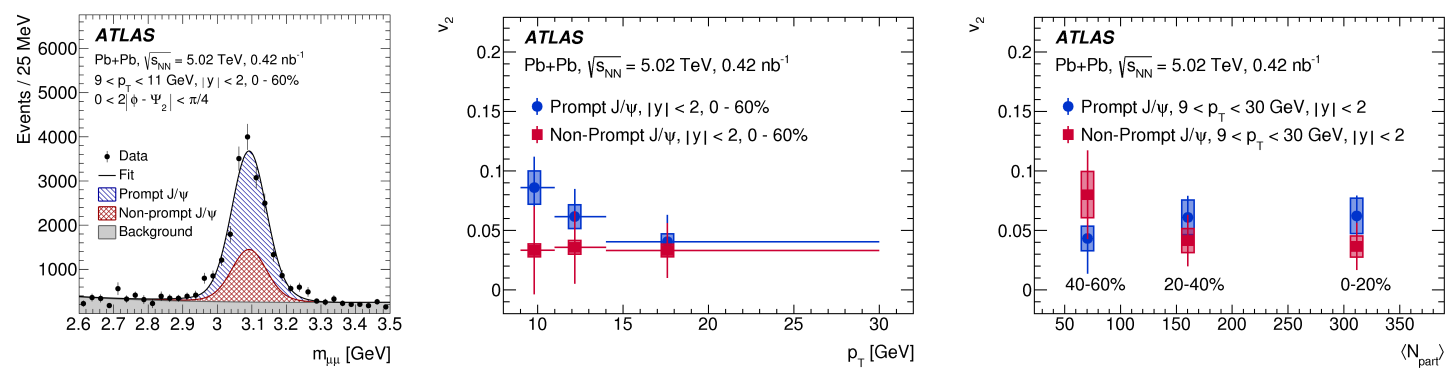

Figure 6: (left) Fit to the di-muon invariant mass for the signal extracted in azimuthal bin $0<2\left|\phi-\Psi_{2}\right|<$ $\pi / 4$. Prompt and non-prompt $J / \psi v_{2}$ as a function of centrality (middle) and $p_{\mathrm{T}}$ (right). The error bars and shaded boxes represent the statistical and systematic uncertainties, respectively. The horizontal error bars in the middle plot represent the kinematic range of the measurement for each bin [12].

The elliptic flow of prompt (c-quarks) and non-prompt (product of $b$-quark decays) $J / \psi$ is measured in the di-muon decay channel. The number of signal candidates and the fraction of 
signal that is non-prompt are extracted from the two-dimensional simultaneous fit of the invariant mass and pseudo-proper decay time of the di-muons from $J / \psi$ candidates. An example plot with fit projection in one azimuthal bin is shown in the left panel of Figure 6. Centrality and $p_{\mathrm{T}}$ dependence of $J / \psi v_{2}$ is shown in Figure 6 for the candidates in the range between $9<p_{\mathrm{T}}<30 \mathrm{GeV}$ and $|\eta|<2$ in $\mathrm{Pb}+\mathrm{Pb}$ collisions at $\sqrt{s_{\mathrm{NN}}}=5.02 \mathrm{TeV}$. The data are consistent with non-zero elliptic flow over the entire studied kinematic range. Prompt $J / \psi v_{2}$ shows decreasing trend with $p_{\mathrm{T}}$ while for nonprompt $J / \psi$ a constant non-zero value of $v_{2}$ is measured with a limited statistical significance. No significant centrality dependence is observed for prompt or non-prompt $J / \psi$ for the results integrated over $p_{\mathrm{T}}$.

\section{Summary}

This report presents recent ATLAS measurements of the flow harmonics in heavy-ion collisions at the LHC. The impact of the centrality fluctuations on multi-particle cumulants and symmetric cumulants is studied in the $\mathrm{Pb}+\mathrm{Pb}$ collisions at $\sqrt{s_{\mathrm{NN}}}=5.02 \mathrm{TeV}$. Results obtained using two reference event classes with different centrality resolution show significant differences in the most central $\mathrm{Pb}+\mathrm{Pb}$ collisions, but a smaller effect is also observed in mid-central collisions. Two new measurements focused on the flow of heavy-quarks are also presented. The first focuses on the flow of the muons originating from semi-leptonic decays of heavy-quarks in $\mathrm{Pb}+\mathrm{Pb}$ collisions at $\sqrt{s_{\mathrm{NN}}}=2.76 \mathrm{TeV}$. The second uses identified $J / \psi$ candidates from $\mathrm{Pb}+\mathrm{Pb}$ collisions at $\sqrt{s_{\mathrm{NN}}}=5.02 \mathrm{TeV}$. Both measurements provide arguments confirming the flow of $c$ - and $b$-hadrons created in heavy-ion collisions. These results provide new and comprehensive information about the nature of the flow of charged particles originating from light- and heavy-quarks.

\section{References}

[1] U. Heinz, R. Snellings, Collective flow and viscosity in relativistic heavy-ion collisions, Annu. Rev. Nucl. Part. Sci. 63 (2013) 123-151, arXiv:1301.2826[nucl-th]

[2] N.Borghini et al., New method for measuring azimuthal distributions in nucleus-nucleus collisions, Phys. Rev. C 63, 054906, arXiv:0007063[nucl-th]

[3] A. Bilandzic et al., Generic framework for anisotropic flow analyses with multi-particle azimuthal correlations, Phys. Rev. C 89, 064904 (2014), arXiv:1313.3572[nucl-th]

[4] ATLAS Collaboration, Correlated long-range mixed-harmonic fluctuations measured in $p p, p+P b$ and low-multiplicity Pb+Pb collisions with the ATLAS detector, Phys. Lett. B 789 (2019) 444, arXiv:1807.02012[nucl-ex]

[5] ATLAS Collaboration, The ATLAS Experiment at the CERN Large Hadron Collider, JINST 3 (2008) S08003

[6] S. Cao, G.-Y. Qin, S. A. Bass, Heavy-quark dynamics and hadronization in ultrarelativistic heavy-ion collisions: Collisional versus radiative energy loss, Phys. Rev. C 88, 044907, arXiv:1308.0617[nucl-ex]

[7] ATLAS Collaboration, Fluctuations of anisotropic flow in Pb+Pb collisions at $\sqrt{s_{N N}}=5.02 \mathrm{TeV}$ with the ATLAS detector, arXiv:1904.04808[nucl-ex] 
[8] M. L. Miller et al., Glauber Modeling in High Energy Nuclear Collisions, Ann. Rev. Nucl. Part. Sci. 57 (2007) 205-243, arXiv:nucl-ex/0701025

[9] B. Alver et al., Importance of correlations and fluctuations on the initial source eccentricity in high-energy nucleus-nucleus collisions, Phys. Rev. C 77 (2008) 014906, arXiv:0711.3724[nucl-ex]

[10] M. Zhou and J. Jia, Centrality fluctuations in heavy-ion collisions, Phys. Rev. C 98 (2018) 044903, arXiv:1803.01812[nucl-ex]

[11] ATLAS Collaboration, Measurement of the suppression and azimuthal anisotropy of muons from heavy-flavor decays in $\mathrm{Pb}+\mathrm{Pb}$ collisions at $\sqrt{s_{N N}}=2.76 \mathrm{TeV}$ with the ATLAS detector, Phys. Rev. C 98 (2018) 044905, arXiv:1805.05220[nucl-ex]

[12] ATLAS Collaboration, Prompt and non-prompt $J / \psi$ and $\psi(2 s)$ suppression at high transverse momentum in $5.02 \mathrm{TeV} \mathrm{Pb+Pb}$ collisions with the ATLAS experiment, Eur. Phys. J. C 78 (2018) 762 , arXiv:1805.04077[nucl-ex]

[13] X. Dong, Y.-J. Lee, and R.Rapp, Open Heavy-Flavor Production in Heavy-Ion Collisions, Annu. Rev. Nucl. Part. Sci. 69 (2019) 417-45, arXiv:1401.3817[nucl-th]

[14] M. He, R. J. Fries, and R. Rapp, Heavy Flavor at the Large Hadron Collider in a Strong Coupling Approach, Phys. Lett. B 735 (2014) 445, arXiv:1903.07709[nucl-ex]

[15] C. A. G. Prado et al., Event-by-event correlations between soft hadrons and $D^{0}$ mesons in $5.02 \mathrm{TeV}$ PbPb collisions at the CERN Large Hadron Collider, Phys. Rev. C 96 (2017) 064903, arXiv:1611.02965[nucl-th] 